\title{
Time is ripe to embrace the scientific approach in Applied Ontology
}

\author{
Stefano Borgo ${ }^{\mathrm{a}, *}$, Pascal Hitzler ${ }^{\mathrm{b}}$ and Cogan Shimizu ${ }^{\mathrm{b}}$ \\ ${ }^{a}$ Laboratory for Applied Ontology, ISTC-CNR, Trento, Italy \\ ${ }^{\mathrm{b}}$ Data Semantics (DaSe) Laboratory, Kansas State University, Manhattan, KS, USA
}

In the 1990s, there has been a rich discussion about how to best understand the term ontology in information science in particular concerning the fact that ontological artifacts should be built and motivated by philosophical principles as defended by Guarino and Giaretta (1995). Today we know that that effort failed to impact the community, as it is widespread habit to use the term ontology to indicate any logical theory that: (a) includes a taxonomy, and (b) is written in a computational (and often decidable) language, such as OWL, the Web Ontology Language (Hitzler et al., 2012). The philosophical view behind the ontology and its actual content have lost centrality except in areas like formal ontology and venues like the FOIS conference series ${ }^{1}$ and the Applied Ontology journal.

There are other topics that were highly debated in the 1990s. One of them, see for instance (Guarino, 1998), is the problem of finding the ideal level of formalization of an ontology. Since a formal ontology is a logical theory about the world and since logic is neutral with respect to the nature and structure of the world ${ }^{2}$ every piece of information about the nature and the structure of the world has to be explicitly coded into a set of logical axioms. This makes the modeling task particularly hard for the ontologist, as one can see comparing ontologies and knowledge representation systems as used in cognitive science (Freksa, 2015).

Another topic of discussion was the proper subdivision of ontologies into types. Guarino (1998) proposed to focus on four types of ontologies: top-level ontologies devoted to "describing very general concepts like space, time, matter, object, event, action, etc." which are independent of a particular problem or domain; domain ontologies that describe the vocabulary related to a generic domain such as medicine or manufacturing; task ontologies that focus on generic tasks or activities such as medical diagnostics or sales; and application ontologies that are devoted to domain-dependent and task-dependent roles, like those of being replaceable or being a spare part ('being replaceable' and 'being a spare part' are roles that objects have in some context or task, they are not essential properties of the objects themselves). This clarification has in part survived, as today many recognize the difference among top-level, domain or reference, and application ontologies. Still, to properly build and use ontologies, the practitioner should know when to classify entities by type versus by role. This has proven to be difficult in practice: once an entity maintains the same role across the relevant scenarios, the theoretical distinction between the entity's type and the entity's role is easily missed, and this jeopardizes cross-application interoperability.

\footnotetext{
${ }^{*}$ Corresponding author: Stefano Borgo, LOA ISTC-CNR, Trento, Italy. E-mail: stefano.borgo@cnr.it.

${ }^{1}$ https://iaoa.org/fois/

${ }^{2}$ One can argue, as it has been done, that logic is not really neutral, in particular regarding our cognitive understanding of the world. This has much to do with what people implicitly mean with the term 'logic'.
} 
Applied ontology is not an isolated research area and from the 1990s has contributed to reshape the context we live in: our decisions and activities are today driven by Artificial Intelligence (AI) algorithms, information is searched using Semantic Web (SW) services, links are HTML connections, and privacy is driven by the open (linked) data approach. Ontology has provided awareness in key points like the differences between linking (an act at the syntactic level) and sharing (an act at the semantic level), between a model (a representation) and a conceptualization (the content of a representation). Yet, applied ontology is still not recognized as a self-standing research domain.

The lack of an established curricula suitable to adequately perform ontological activities has certainly hurt. Activities in applied ontologies are definitely complex as they require acquaintance with several domains and techniques from ontological analysis to logical modeling, from knowledge-base building to customization. However, the lack of recognized curricula is not entirely the problem: it matches the lack of benchmarks to establish satisfactory levels of ontological systems, and of recognized methodologies to compare ontologies in terms of their quality or modeling capabilities (Neuhaus et al., 2013). It is natural to ask what is holding back the community from developing these benchmarks and methodologies.

There are recognized methodologies in applied ontology, spanning all four of the ontology types and a broad range of applications:

- How to build a taxonomy (Guarino and Welty, 2009).

- How to build a whole ontology from scratch (Suárez-Figueroa et al., 2015; Spyns et al., 2008).

- How to build ontologies by reusing ontology design patterns in a modular manner (Blomqvist et al., 2016; Shimizu et al., 2020b).

But why did the community stop here?

We have argued elsewhere (Borgo and Hitzler, 2018) that most foundational ontologies share their core assumptions, but argue about their philosophical comprehension: the identity criteria of objects, the existence of abstracts, the ontological nature of events, and the understanding of properties. Are these differences relevant to the construction of ontological systems, as needed in applied ontology? We do not know because this question has not attracted enough attention yet.

Perhaps we should start asking how contemporary work is carried out in applied ontology, broadly understood. In the practice of technological and application fields, we notice that much work, that uses the label ontology, can be described as a translation of existing models into modern formal languages, such as OWL. On the one hand, this means that these works are bound to representational choices made in the last century with modeling awareness of those times.

At a slightly more sophisticated level, ontological research is aiming at explicitly establishing a unique match between a term and a (technical) meaning. This systematic revision of terminology leads to overcome the ambiguities still present in today's descriptions and models. When the ontologist limits herself to this aim, ontological activity can be described as vocabulary construction, and ontology as modeling without semantic ambiguities. This is an important goal, of course, but is applied ontology just this?

At an even higher level, applied ontology is a branch of philosophy, where the philosophical principles are spelled out in formal terms and a general viewpoint of reality is captured in logical forms.

However interesting these levels can be, none of them seem to present the gist of applied ontology. Applied ontology is not just (philosophical) ontology and is not just conceptual clarity. Applied ontology has been thought as a solution to scientific problems. As such, it must be a scientific enterprise. What is the scientific advancement of translating old models into modern formats? What is the scientific achievement of having a well-set vocabulary? What is the scientific relevance of having a formalization of a philosophical position? 
A scientific result is obtained by making hypotheses, building theories, applying them, and making comparisons. The competition among theories and systematic experimentation are key to science. Where is the latter in today's research in applied ontology? Where is the comparison of different model capabilities? The analysis of the practical suitability of a theory for a domain? Even when there is a chance to start this work, see the recent IOF effort, ${ }^{3}$ participants prefer to go ahead without attempting to test and compare ontologies for their capabilities to model and capture the information needs of the targeted domain. We believe that this attitude is at the core of the shortcomings of applied ontology today and should be abandoned.

Perhaps there are two issues in applied ontology which cannot be overestimated: expressivity needs and hidden assumptions. The development of logical theories is an important and necessary part of applied ontology. We know that much effort has been put into developing formal theories of notions like parthood and dependence, which are rarely considered in their full generality in logical studies. Ontologists have tried to make the best out of formalisms created for other purposes (e.g. foundation of mathematics, theoretical computer science, or natural language modeling). Attempts to introduce operators that are ontologically motivated, e.g. as in (Borgo et al., 2014), are scarce. This means that today it is hard to identify the expressivity needs of a domain, even though this is a fundamental step in any ontological project.

The study of philosophical theories has led to identify many assumptions that are implicitly made in a conceptualization of reality. These must be paired with the study of the assumptions, often hidden, made in the domain at stake. Besides being a fundamental step for ontological research, knowing the expressivity needs and the implicit assumptions open the way to ontologically evaluate and possibly rethink an entire domain. This can lead to a revolution in model construction and problem solving, a result that is achievable if we recall that the term 'applied' in applied ontology is not a qualifier but a modifier: ontology is a philosophical discipline, applied ontology is a scientific one.

Of course, none of these issues is simple. The availability of software tools developed for ontology can help in a number of different ways. The development of an ontology, of any type, is an extraordinarily expensive effort. It requires a high time commitment from ontologists, domain experts, and software developers in order to implement an entire application. Of course, when any methodology is difficult to execute, the science slows down-especially when that science requires many such executions. Improving the surrounding tool infrastructure can both reduce the amount of time needed to iterate a methodology, but also reduce the amount of effort any particular execution may take.

There already exist some formalisms that help during the modeling processes. For instance, DOL (Mossakowski et al., 2015) allows the connection of different representation languages to create a unified whole. This is less of a tool in a software sense, but a tool in the form that facilitates interdisciplinary modeling. In terms of software improvements, we could consider ontology editors (e.g. Stanford's Protégé ${ }^{4}$ ) that help the authoring of ontologies. In particular, we reference a plug-in for that platform, called CoModIDE, which enables graphical modeling, and has been shown to speed up the modeling process, especially for those who are not ontologists (Shimizu et al., 2020a).

However, we see further needs for tool support. For instance, to: (a) test different combinations of principles (via their logical formalization) and verify modeling capabilities; (b) weaken the dependence of ontological theories on philosophical stands to better concentrate on clarity and interoperability; (c) test different ontologies in the same modeling task obtaining comparison assessment and leading to select

\footnotetext{
${ }^{3}$ https://sites.google.com/view/industrialontologies/home

${ }^{4}$ https://protege.stanford.edu/
} 
and tune the most suitable ontology; (d) integrate results from linguistics and cognitive science to test and enhance transparency and communication capacities.

Tools that provide these capacities would go a long way to improving the state of applied ontology by allowing for quick and easy, multifaceted, critical comparison of ontological approaches and commitments.

We believe that success in applied ontology must be understood as a scientific, not a philosophical term. Success of an ontology means that with it knowledge is correctly modeled (both practically and philosophically) and that superiority is verified via practical comparisons in the domain. This is not happening yet. A scientific turn is needed and we believe that time is ripe for applied ontology to finally become a scientific discipline. A chance to investigate this might come again from a European project. The first formal foundational ontology has been the outcome of the WonderWeb project ${ }^{5}$ in 2002 , it is likely that another European project, now at its preparatory stage and planned to start in fall $2020,{ }^{6}$ will give us the first scientific analysis and comparison on the application field of different ontological systems. After twenty years of ontology construction, it is time to start comparing!

Notwithstanding these big challenges ahead, the field continues to progress. This special issue collects four papers from the Formal Ontology in Information Systems (FOIS) Conference in 2018 that were selected for their quality, the overall approach, and the achieved results. These works improve our understanding of the world, as well as provide useful resources to emerging domains. In particular, the paper "SAREF4health: Towards IoT Standard-Based Ontology-Driven Cardiac e-Health Systems" focuses on semantic interoperability of IoT solutions and received the FOIS Best Paper Award witnessing the interest of the community towards the application of formal ontology in application scenarios. The paper "The FOUnt Ontologies for Quantities, Units, and the Physical World" addresses one of the points we discussed above, the need to understand hidden assumptions (in this case in the use of quantities), and shows how to rethink the connection between quantities, units and processes. The paper "CODI: A Multidimensional Theory of Mereotopology with Closure Operations" investigates the advantages of using multidimensional mereotopology for geometric data models (like those in GIS, CAD, and CAM) for capturing both qualitative and quantitative knowledge. Finally, the paper "Parthood and part-whole Relations in Zulu Language and Culture" analyzes the use of mereological relations in the isiZulu language, raising questions on how to manage the plurality of relations exemplified in application domains, and on how the accepted view of mereology can cope with this proliferation of relations.

We would like to thanks the authors for their contributions and the dedication of our reviewers (Robert Hoehndorf, Ludger Jansen, Till Mossakowski, and Antoine Zimmerman) that helped to improve further the quality of this special issue.

\section{References}

Blomqvist, E., Hammar, K. \& Presutti, V. (2016). Engineering ontologies with patterns - The eXtreme design methodology. In P. Hitzler, A. Gangemi, K. Janowicz, A. Krisnadhi and V. Presutti (Eds.), Ontology Engineering with Ontology Design Patterns - Foundations and Applications. Studies on the Semantic Web (Vol. 25, pp. 23-50). IOS Press. doi:10.3233/9781-61499-676-7-23.

\footnotetext{
${ }^{5}$ https://cordis.europa.eu/project/id/IST-2001-33052

${ }^{6}$ The project "Ontology-driven data documentation for Industry Commons (OntoCommons)" has been selected for funding in call dt-nmbp-39-2020, see https://ec.europa.eu/info/funding-tenders/opportunities/portal/screen/opportunities/topic-details/ dt-nmbp-39-2020.
} 
Borgo, S. \& Hitzler, P. (2018). Some open issues after twenty years of formal ontology. In Formal Ontology in Information Systems. Frontiers in Artificial Intelligence and Applications (Vol. 306, pp. 1-9). IOS Press. doi:10.3233/978-1-61499-910$2-1$.

Borgo, S., Porello, D. \& Troquard, N. (2014). Logical operators for ontological modeling. In O. Kutz and P. Garbacz (Eds.), FOIS 2014 - Formal Ontology in Information Systems. Frontiers in Artificial Intelligence and Applications (Vol. 267, pp. 23-36). IOS Press.

Freksa, C. (2015). Strong spatial cognition. In International Conference on Spatial Information Theory (pp. 65-86). Springer. doi:10.1007/978-3-319-23374-1_4.

Guarino, N. (1998). Formal ontology in information systems. In N. Guarino (Ed.), Proceedings of the Second International Conference on Formal Ontology in Information Systems (pp. 3-15). IOS Press.

Guarino, N. \& Giaretta, P. (1995). Ontologies and knowledge bases: Towards a terminological clarification. In N. Mars (Ed.), Towards Very Large Knowledge Bases: Knowledge Building and Knowledge Sharing (pp. 25-32). Amsterdam: IOS Press.

Guarino, N. \& Welty, C. (2009). An overview on OntoClean. In S. Staab and R. Studer (Eds.), Handbook on Ontologies (2nd ed., pp. 201-220). Springer. doi:10.1007/978-3-540-92673-3_9.

Hitzler, P., Krötzsch, M., Parsia, B., Patel-Schneider, P.F. \& Rudolph, S. (Eds.) (2012). OWL 2 web ontology language: Primer (second edition). W3C Recommendation 11 December 2012. Available from http://www.w3.org/TR/owl2-primer/.

Mossakowski, T., Codescu, M., Neuhaus, F. \& Kutz, O. (2015). The distributed ontology, modeling and specification language - DOL. In The Road to Universal Logic (pp. 489-520). Springer. doi:10.1007/978-3-319-15368-1_21.

Neuhaus, F., Vizedom, A., Baclawski, K., Bennett, M., Dean, M., Denny, M., Grüninger, M., Hashemi, A., Longstreth, T., Obrst, L., et al. (2013). Towards ontology evaluation across the life cycle. Applied Ontology, 8(3), 179-194. doi:10.3233/ AO- 130125.

Shimizu, C., Hammar, K. \& Hitzler, P. (2020a). Modular graphical ontology engineering evaluated. In A. Harth, S. Kirrane, A.N. Ngomo, H. Paulheim, A. Rula, A.L. Gentile, P. Haase and M. Cochez (Eds.), The Semantic Web - 17th International Conference, ESWC 2020, Heraklion, Crete, Greece, May 31-June 4, 2020, Proceedings. Lecture Notes in Computer Science (Vol. 12123, pp. 20-35). Springer. doi:10.1007/978-3-030-49461-2_2.

Shimizu, C., Krisnadhi, A. \& Hitzler, P. (2020b). Modular ontology modeling: A tutorial. In G. Cota, M. Daquino and G.L. Pozzato (Eds.), Applications and Practices in Ontology Design, Extraction, and Reasoning. Studies on the Semantic Web. IOS Press. To appear.

Spyns, P., Tang, Y. \& Meersman, R. (2008). An ontology engineering methodology for DOGMA. Applied Ontology, 3(1-2), 13-39. doi:10.3233/AO-2008-0048.

Suárez-Figueroa, M.C., Góómez-Perez, A. \& Fernández-López, M. (2015). The NeOn methodology framework: A scenariobased methodology for ontology development. Applied Ontology, 10, 107-145. doi:10.3233/AO-150145. 